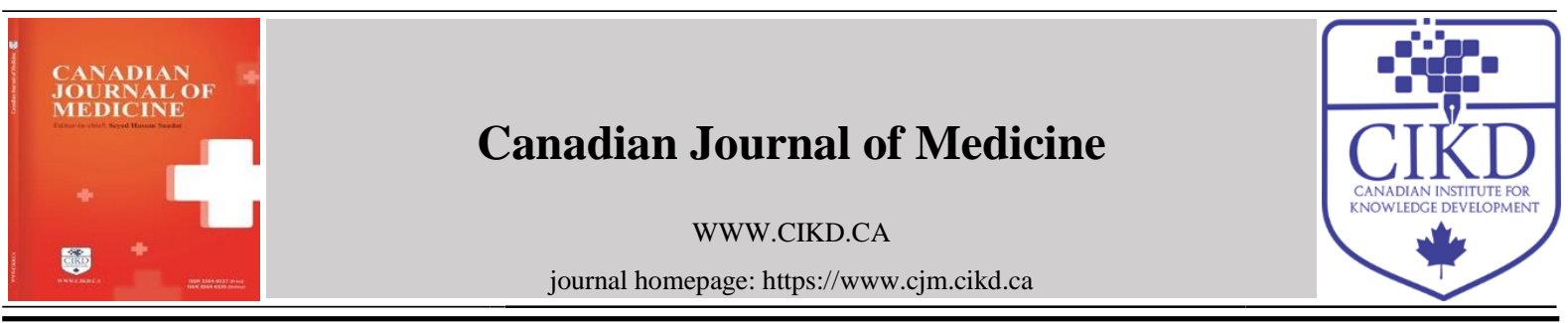

Review

\title{
How will the COVID-19 Pandemic Change Dermatology Services over the next Five Years?
}

\author{
Immanuel Sani*1 ${ }^{*}$ Damilola Agboluaje ${ }^{2}$ \\ ${ }^{1}$ Leicester Medical School, George Davies Centre, Lancaster Road, Leicester LE1 7HA, United \\ Kingdom \\ ${ }^{2}$ King's College London, GKT School of Medical Education, London
}

\begin{abstract}
Keywords:

Dermatology, COVID-19,

Innovation, Primary care

\section{Received}

04 August 2021

Received in revised form

07 November 2021

Accepted

11 November 2021

*Correspondence:

is197@student.le.ac.uk

The advent of COVID-19 has radically transformed conventional affairs in numerous facets of life across the world. The reverberation of such alterations has presented a myriad of challenges to dermatology services worldwide. Dermatology services have attempted to suppress the dissemination of COVID-19 by reducing in-person consultations and nonessential procedures. Teledermatology has been utilised to mediate patient triage to ensure patients are promptly referred to the appropriate service. Additionally, a plethora of cutaneous sequelae of COVID-19 have been identified and exhibit considerable heterogeneity in skin inflammatory findings compared to viral infections with known cutaneous effects. There has been a longstanding demand to efficiently capitalise on limited expertise allied to dermatology services. The COVID-19 pandemic has illuminated the urgent need to extend the dermatological competence of several primary care clinicians. Ultimately, the developing COVID-19 pandemic may provide the impetus to revolutionise dermatology services in the next five years to transcend current challenges in clinical practice.
\end{abstract}

CCIKD Publishing

The advent of COVID-19 has radically transformed conventional affairs in numerous facets of life across the world. The reverberation of such alterations has presented a myriad of challenges to dermatology services worldwide [1]. Given that, $20 \%$ of general physician (GP) consultations are related to skin disease, representing a 54\%-point prevalence per annum [2]. The increasing pressure of the COVID-19 pandemic on dermatology services warrants interventional strategies that will address ongoing and emerging challenges in dermatology amidst the current climate, with the intent of driving innovation within the service. Hence, this 
manuscript will discuss the impact of COVID-19 on dermatology patient triage, current knowledge on the cutaneous manifestation of COVID-19 infection and application to the management of recalcitrant dermatitis, and the dearth of dermatology specialists and healthcare personnel as well as exploring measures that may reform dermatology services over the next five years concerning the aforementioned challenges in question.

\section{Dermatology Services}

This part has attempted to suppress the dissemination of COVID-19 by reducing in-person consultations and non-essential procedures characterised by $53.4 \%$ and $74.4 \%$ decrement in both operations, respectively [3]. Teledermatology, which involves the transfer of an image of the affected integumentary component of a patient coupled with a relevant history of presenting complaints to a clinician for advice, has since been utilised three-fold beyond pre-COVID-19 frequency [3]. This approach has posed utility in mediating patient triage to ensure patients are promptly referred to the appropriate service. Teledermatology in primary care may also enhance the productivity of clinicians by virtue of reduced travel, enhance patient access to care, and lead to significant cost savings [4]. Such cost savings can be derived from offsetting patient or clinicians' travel to clinics financed by a public healthcare system. Considering these benefits, it is, therefore, logical to suggest that teledermatology may positively change the landscape of dermatology in the next five years in tandem with the exponential advancement in digital technology. Future innovations in the dermatology triage service should encompass real-time diagnostic data transfer and secure, private cloud technology for swift intra- and interprofessional communication and coordination of patient care.

\section{Dermatology Challenge}

Another unprecedented challenge in current dermatology practice pertains to the assessment and management of COVID-19 secondary dermatitis. A plethora of cutaneous sequelae of COVID-19 has been identified and exhibited considerable heterogeneity in skin inflammatory findings compared to viral infections with known cutaneous effects. For instance, the presence of chilblains-like lesions amongst the symptomatology of COVID-19 raises the question of a potential delayed immune response as the aetiology of such lesions [5]. Furthermore, the causality of chilblains-like lesions in COVID-19 remains controversial as this symptom may arise owing to pre-existing systemic diseases that serves as risk factors for developing COVID19 rather than mere correlation. To that end, in the next five years, dermatology services must endeavour to conduct rigorous, prospective research on the cause and natural trajectory of COVID-19-associated dermatopathology as well as identification of effective management strategies. Attainment of this knowledge may also be extrapolated to comparable inflammatory skin conditions, with limited evidence, to further improve the quality of care offered by dermatology in rare dermatologic cases that necessitate multidisciplinary management.

There has been a longstanding demand to efficiently capitalise on limited expertise allied to dermatology services. The COVID-19 pandemic has illuminated the urgent need to extend the dermatological competence of several primary care clinicians. Indeed, dermatology constitutes an insignificant portion of GP training, which results in a deficiency in dermatologic diagnostic capabilities [6]. Dermatology services should implement greater utilisation of GPs with special interest (GPwSI) and specialist nurses to partition the overall workload burden on consultant 
dermatologists. GPwSI particularly may be crucial in providing subsequent patient care, where specialist input is not required and can therefore be capable of improving the transit of patients through dermatology services. However, GPwSI must work cohesively with specialists to uphold the quality of patient care and reduce the frequency of unnecessary referrals. As such, efforts should be made by dermatology services and medical schools alike to increase medical students' exposure to the role of GPwSI to raise interest in this role and develop a larger future workforce in this domain.

\section{Conclusion}

The developing COVID-19 pandemic may provide the impetus to revolutionise dermatology services in the next five years to transcend current challenges in clinical practice. This manuscript aimed to provide an encouraging outlook on the future of dermatology and to draw attention to potentially effective measures that may improve patient care and safety offered by dermatology services in the near future when be employed consistently and in combination with existing measures.

\section{References}

[1] Darlenski R, Tsankov R. "COVID-19 pandemic and the skin: what should dermatologists know?." Clinics in dermatology. (2020).38(6):785-787. doi:10.1016/j.clindermatol.2020.03.012.

[2] Schofield JK, Grindlay D, William HC. Skin conditions in the UK: a health needs assessment. 2009. Available from: www.nottingham.ac.uk/scs/divisions/evidencebased dermatology/news/dermatologyhealthcareneeds assessmentreport.aspx

[3] Bhargava S, McKeever C, Kroumpouzos G. Impact of covid-19 pandemic on dermatology practice: results of a webbased, global survey. Int J Womens Dermatol. 2020;10.

[4] Snoswell CL, Taylor ML, Comans TA, Smith AC, Gray LC, Caffery LJ. Determining if telehealth can reduce health system costs: Scoping Review. J Med Internet Res 2020;22(10):e17298.

[5] Galván CC, Català A, Carretero HG, et al. Classification of the cutaneous manifestations of COVID-19: a rapid prospective nationwide consensus study in Spain with 375 cases. Br J Dermatol. 2020;183(1):71-77.

[6] The King's Fund. How can dermatology services meet current and future patient needs, while ensuring quality of care is not compromised and access is equitable across the UK? 2014.

\section{Acknowledgements}

Not applicable.

\section{Disclosure Statement}

No potential conflict of interest was reported by the authors.

\section{Funding Acknowledgements}

Not applicable. 